\title{
Tolvaptan-induced hypernatremia related to low serum potassium level accompanying high blood pressure in patients with acute decompensated heart failure
}

Hidetada Fukuoka, Koichi Tachibana, Yukinori Shinoda, Tomoko Minamisaka, Hirooki Inui, Keisuke Ueno, Soki Inoue, Kentaro Mine, Kumpei Ueda and Shiro Hoshida* (1)

\begin{abstract}
Backgrounds: Tolvaptan significantly increases urine volume in acute decompensated heart failure (ADHF); serum sodium level increases due to aquaresis in almost all cases. We aimed to elucidate clinical factors associated with hypernatremia in ADHF patients treated with tolvaptan.

Methods: We enrolled 117 ADHF patients treated with tolvaptan in addition to standard therapy. We examined differences in clinical factors at baseline between patients with and without hypernatremia in the initial three days of hospitalization.

Results: Systolic $(p=0.045)$ and diastolic $(p=0.004)$ blood pressure, serum sodium level $(p=0.002)$, and negative water balance $(p=0.036)$ were significantly higher and serum potassium level $(p=0.026)$ was significantly lower on admission day in patients with hypernatremia $(n=22)$. In multivariate regression analysis, hypernatremia was associated with low serum potassium level $(p=0.034)$. Among patients with serum potassium level $\leq 3.8 \mathrm{mEq} / \mathrm{L}$, the cutoff value obtained using receiver operating characteristic curve analysis, those with hypernatremia related to tolvaptan treatment showed significantly higher diastolic blood pressure on admission day $(p=0.004)$.
\end{abstract}

Conclusion: In tolvaptan treatment combined with standard therapy in ADHF patients, serum potassium level $\leq 3.8 \mathrm{mEq} / \mathrm{L}$ may be a determinant factor for hypernatremia development. Among hypokalemic patients, those with higher diastolic blood pressure on admission may be carefully managed to prevent hypernatremia.

Keywords: Acute decompensated heart failure, Hypernatremia, Hypokalemia, Tolvaptan

\section{Backgrounds}

Tolvaptan, a selective V2 receptor antagonist with an aquaretic effect, significantly increases urine volume without increasing electrolyte excretion into the urine in acute decompensated heart failure (ADHF) [1-3]. Tolvaptan can decrease body weight, increase serum

*Correspondence: shiro.hoshida@hosp-yao.osaka.jp Department of Cardiovascular Medicine, Yao Municipal Hospital, 1-3-1 Ryuge-cho, Yao, Osaka 581-0069, Japan sodium level, and ameliorate some congestion symptoms in patients with ADHF, which may help prevent overdose of loop diuretics, especially in patients with renal dysfunction [4]. A meta-analysis of the published literature suggests short-term benefits of tolvaptan, but the impact on mortality is inconclusive [4-7]. The serum sodium level increases as a result of aquaresis in almost all cases, and hypernatremia can be lethal in some patients $[8,9]$ and was identified as a significant adverse event to be prevented [10]. Therefore, a lower dose of tolvaptan to 
prevent hypernatremia has been recommended in the initial phase [11, 12], because tolvaptan treatment can dose dependently lead to abnormal hypernatremia [13, 14]. Sometimes, hypernatremia results in central nervous system disturbance. There is a population that is a risk to the development of hypernatremia [15], and risk factors for hypernatremia in tolvaptan treatment were previously reported [10-12]. This study aimed to elucidate clinical factors associated with hypernatremia in patients with ADHF treated with full medications and tolvaptan in real-world practice.

\section{Methods Subjects}

We retrospectively investigated 117 consecutive inhospital patients with ADHF (mean age, 78 years) who received oral tolvaptan therapy in addition to standard therapy, including carperitide infusion, for the treatment of volume overload between January 2016 and December 2018 in our cardiology ward. Heart failure (HF) symptoms in all patients worsened despite treatment including oral diuretic therapy before hospital admission. Patients were excluded if they had anuria, consciousness disturbance, and cardiogenic shock.

\section{Procedure}

All patients underwent baseline blood and urine tests, including neurohumoral assessment such as plasma B-type natriuretic peptide (BNP), renin activity, and aldosterone concentration, chest X-rays, and echocardiography on admission day. Serum osmolality was calculated using the following equation:

\section{Classification of hypernatremia}

The development of hypernatremia was defined in a risk analysis when at least one measurement of serum sodium level was $\geq 148 \mathrm{mEq} / \mathrm{L}$ in the initial three days after tolvaptan treatment. Predictive factors that affect the development of hypernatremia by tolvaptan treatment were extracted from variables in clinical characteristics, blood and urine tests, and medications.

\section{Statistical analysis}

All numerical data are expressed as mean \pm standard deviation or percentages. Continuous data were compared using the unpaired t-test. Categorical data were assessed using the chi-square test. The area under the curve was calculated, and optimal cutoff values of predictors of hypernatremia were determined. A multivariate logistic regression analysis was applied to assess the independent factors showing hypernatremia using the variables that were significant in the univariate analysis. $p$ values $<0.05$ were considered statistically significant. All statistical analyses were performed using EZR (Saitama Medical Center, Jichi Medical University, Saitama, Japan), which is a graphical user interface for $\mathrm{R}$ (The $\mathrm{R}$ Foundation for Statistical Computing, Vienna, Austria).

\section{Results}

Baseline characteristics in patients with hypernatremia Systolic $(p=0.045)$ and diastolic $(p=0.004)$ blood pressures were significantly higher on admission day in patients with hypernatremia $(\mathrm{n}=22$, Table 1$)$. However, no differences were observed in comorbidities, such as diabetes, hypertension, and dyslipidemia, and medications before admission between patients with and without hypernatremia. The incidence of atrial fibrillation

Calculated serum osmolality $=2 \times \mathrm{Na}+$ blood urea nitrogen $/ 2.8+$ blood sugar $/ 18$.

Vital signs, 24-h fluid intake, and urine volume were measured at baseline and every $24 \mathrm{~h}$ thereafter. Body weight was measured after urination and before breakfast at baseline. First-morning spot urine tests included the measurements of osmolality and sodium (UNa), potassium, urea nitrogen (UUN), and creatinine (UCr) levels. The following formula was used to estimate urine osmolality:

$$
\text { Urine osmolality }=1.07 \times\{2 \times[\mathrm{UNa}(\mathrm{mEq} / \mathrm{L})]+[\mathrm{UUN}(\mathrm{mg} / \mathrm{dL})] / 2.8+[\mathrm{UCr}(\mathrm{mg} / \mathrm{dL})] \times 2 / 3\}+16 .
$$

was also not different (Table 1). Regarding laboratory data, there were no differences in BNP level; estimated glomerular filtration rate; albumin, blood sugar, and uric acid levels; renin activity; and aldosterone level between the two groups (Table 1). However, serum sodium level $(p=0.002)$ was significantly higher, and serum potassium level $(p=0.026)$ was significantly lower at baseline in patients with hypernatremia (Table 1). We did
It was planned that all patients would undergo repeated blood and urine tests during 3 days after admission. Left ventricular ejection fraction was assessed by echocardiography using the biplane Simpson's rule. not observe differences in urine examination results at baseline. When we calculated serum osmolality by sodium, blood urea nitrogen, and blood sugar levels, patients exhibiting hypernatremia showed significantly 
Table 1 Baseline characteristics of patients on admission day with and without hypernatremia in the initial three days after tolvaptan treatment

\begin{tabular}{|c|c|c|c|}
\hline & With hypernatremia & Without hypernatremia & $p$ value \\
\hline N (\%) & $22(19)$ & $95(81)$ & \\
\hline Age, years & $78.5 \pm 12.2$ & $77.3 \pm 11.3$ & 0.661 \\
\hline Men, \% & 46 & 57 & 0.337 \\
\hline Body weight, kg & $57.9 \pm 13.8$ & $58.6 \pm 20.3$ & 0.850 \\
\hline Body mass index, kg/m² & $23.9 \pm 6.3$ & $23,4 \pm 4.0$ & 0.633 \\
\hline LVEF, \% & $44 \pm 18$ & $45 \pm 19$ & 0.869 \\
\hline Systolic blood pressure, mmHg & $149 \pm 21$ & $137 \pm 25$ & 0.045 \\
\hline Diastolic blood pressure, $\mathrm{mmHg}$ & $90 \pm 20$ & $78 \pm 17$ & 0.004 \\
\hline Heart rate, beats/min & $95 \pm 24$ & $87 \pm 23$ & 0.152 \\
\hline \multicolumn{4}{|l|}{ NYHA, class } \\
\hline | or II, \% & 77 & 55 & 0.089 \\
\hline III or IV, \% & 23 & 45 & \\
\hline \multicolumn{4}{|l|}{ Medical history } \\
\hline Diabetes mellitus, \% & 36 & 42 & 0.623 \\
\hline Hypertension, \% & 73 & 64 & 0.452 \\
\hline Dyslipidemia, \% & 32 & 37 & 0.661 \\
\hline Atrial fibrillation, $\%$ & 55 & 43 & 0.338 \\
\hline Coronary artery disease, $\%$ & 23 & 25 & 0.806 \\
\hline Valvular disease, $\%$ & 32 & 27 & 0.679 \\
\hline Cardiomyopathy, \% & 5 & 11 & 0.391 \\
\hline \multicolumn{4}{|l|}{ Medications before admission } \\
\hline ACEI/ARB, $\%$ & 41 & 39 & 0.867 \\
\hline$\beta$-blocker, $\%$ & 55 & 40 & 0.217 \\
\hline Ca channel blocker, \% & 50 & 39 & 0.347 \\
\hline Loop diuretics, \% & 45 & 53 & 0.548 \\
\hline MRA, \% & 23 & 13 & 0.230 \\
\hline Thiazide, $\%$ & 0 & 7 & 0.192 \\
\hline \multicolumn{4}{|l|}{ Laboratory data at baseline } \\
\hline $\mathrm{BNP}, \mathrm{pg} / \mathrm{mL}$ & $1043 \pm 758$ & $1127 \pm 992$ & 0.711 \\
\hline Hematocrit, $\%$ & $35.3 \pm 8.2$ & $34.7 \pm 7.5$ & 0.722 \\
\hline Albumin, $\mathrm{g} / \mathrm{dL}$ & $3.9 \pm 0.5$ & $3.7 \pm 0.5$ & 0.182 \\
\hline Blood urea nitrogen, mg/dL & $27.2 \pm 19.8$ & $27.5 \pm 14.4$ & 0.931 \\
\hline Serum creatinine, mg/dL & $1.8 \pm 2.3$ & $1.3 \pm 0.8$ & 0.110 \\
\hline $\mathrm{eGFR}, \mathrm{mL} / \mathrm{min} / 1.73 \mathrm{~m}^{2}$ & $44.6 \pm 20.9$ & $46.6 \pm 18.6$ & 0.654 \\
\hline Uric acid, mg/dL & $6.5 \pm 2.4$ & $6.4 \pm 2.3$ & 0.817 \\
\hline Serum sodium, mEq/L & $143 \pm 3$ & $140 \pm 4$ & 0.002 \\
\hline Serum potassium, mEq/L & $3.9 \pm 0.5$ & $4.3 \pm 0.6$ & 0.026 \\
\hline Blood sugar, mg/dL & $145 \pm 75$ & $141 \pm 57$ & 0.812 \\
\hline Serum osmolality, mOsm/L & $295 \pm 9$ & $291 \pm 11$ & 0.139 \\
\hline \multicolumn{4}{|l|}{ Hormone at baseline } \\
\hline $\mathrm{PRA}, \mathrm{ng} / \mathrm{mL} / \mathrm{h}$ & $1.0 \pm 1.5$ & $2.7 \pm 4.7$ & 0.204 \\
\hline $\mathrm{PAC}, \mathrm{pg} / \mathrm{mL}$ & $93 \pm 108$ & $117 \pm 170$ & 0.631 \\
\hline Adrenaline, pg/mL & $75 \pm 138$ & $58 \pm 133$ & 0.690 \\
\hline Noradrenaline, pg/mL & $1059 \pm 723$ & $709 \pm 985$ & 0.234 \\
\hline Dopamine, pg/mL & $35 \pm 27$ & $47 \pm 140$ & 0.753 \\
\hline \multicolumn{4}{|l|}{ Urine examination at baseline } \\
\hline Urine urea nitrogen, mg/dL & $478 \pm 397$ & $478 \pm 405$ & 0.999 \\
\hline Urine creatinine, mg/dL & $71.3 \pm 64.9$ & $86.0 \pm 90.8$ & 0.579 \\
\hline Urine sodium, mEq/L & $84.4 \pm 42.8$ & $91.9 \pm 50.1$ & 0.546 \\
\hline Urine potassium, mEq/L & $27.0 \pm 18.4$ & $29.7 \pm 26.3$ & 0.733 \\
\hline Urine osmolality, mOsm/L & $450 \pm 182$ & $432 \pm 188$ & 0.707 \\
\hline
\end{tabular}

NYHA New York Heart Association, $A C E l$ angiotensin-converting enzyme inhibitor, ARB angiotensin receptor blocker, MRA mineralocorticoid receptor antagonist, $B N P$ brain natriuretic peptide, eGFR estimated glomerular filtration rate, PRA plasma renin activity, PAC plasma aldosterone concentration 
Table 2 Calculated parameters at baseline in patients with and without hypernatremia

\begin{tabular}{lcll}
\hline & With hypernatremia & $\begin{array}{l}\text { Without } \\
\text { hypernatremia }\end{array}$ & p value \\
\hline BUN/Cr & $19.2 \pm 7.6$ & $22.5 \pm 7.7$ & 0.074 \\
PAC/PRA & $144 \pm 144$ & $142 \pm 158$ & 0.969 \\
C-serum osmo- & $304 \pm 7$ & $298 \pm 10$ & 0.012 \\
lality, mOsm/L & & & \\
U-Osm/S-Osm & $1.5 \pm 0.6$ & $1.5 \pm 0.7$ & 0.838 \\
FENa, \% & $2.3 \pm 2.5$ & $3.3 \pm 4.8$ & 0.434 \\
FEUN, \% & $36.8 \pm 12.9$ & $34.8 \pm 12.6$ & 0.623 \\
FEK, \% & $18.1 \pm 18.3$ & $15.1 \pm 10.4$ & 0.442 \\
TTKG & $4.3 \pm 1.7$ & $4.3 \pm 2.0$ & 0.995 \\
\hline
\end{tabular}

$\mathrm{C}$-Serum osmolality $=2 \times \mathrm{Na}+\mathrm{BUN} / 2.8+$ blood sugar $/ 18 ; \mathrm{FENa}=(\mathrm{U}-\mathrm{Na} \times \mathrm{S}-\mathrm{Cr}) /$ $(\mathrm{U}-\mathrm{Cr} \times \mathrm{S}-\mathrm{Na}) \times 100 ; \mathrm{FEUN}=(\mathrm{U}-\mathrm{UN} \times \mathrm{S}-\mathrm{Cr}) /(\mathrm{U}-\mathrm{Cr} \times \mathrm{S}-\mathrm{UN}) \times 100$;

$\mathrm{FEK}=(\mathrm{U}-\mathrm{K} \times \mathrm{S}-\mathrm{Cr}) /(\mathrm{U}-\mathrm{Cr} \times \mathrm{S}-\mathrm{K}) \times 100 ; \mathrm{TTKG}=\{\mathrm{U}-\mathrm{K} /(\mathrm{U}-\mathrm{Osm} / \mathrm{S}-\mathrm{Osm})\} / \mathrm{S}-\mathrm{K}$

$U$ - urine-, $S$ - serum-, $C$ - calculated, $B U N / C r$ ratio of blood urea nitrogen to serum creatinine, $P A C / P R A$ ratio of plasma aldosterone concentration to plasma renin activity, U-Osm/S-Osm ratio of urine osmolality to serum osmolality, FENa fractional excretion of sodium, FEUN fractional excretion of urea nitrogen, FEK fractional excretion of potassium, TTKG trans-tubular K gradient

higher calculated serum osmolality $(p=0.012$, Table 2$)$. There were no differences in the doses of tolvaptan $(7.5 \pm 3.8$ vs. $8.1 \pm 2.5 \mathrm{mg} /$ day, $p=0.269)$ and carperitide $(0.025 \pm 0.010$ vs. $0.025 \pm 0.06 \mu \mathrm{g} / \mathrm{min}, p=0.835)$ between patients with and without hypernatremia.

Regarding water balance calculated using the equation of (urine volume-water intake), dehydration obviously occurred during the first hospitalization day in patients with hypernatremia $(p=0.036$, Table 3$)$. In the multivariate regression analysis using significant factors observed in the univariate analysis, hypernatremia in the initial three days of hospitalization was independently associated with low serum potassium level $(p=0.034$, Table 4$)$. The cutoff serum potassium level at baseline was $3.8 \mathrm{mEq} / \mathrm{L}$ by the receiver operating characteristic curve analysis (Fig. 1).
Table 4 Multivariate regression analysis of factors predicting hypernatremia

\begin{tabular}{|c|c|c|c|}
\hline & OR & $95 \% \mathrm{Cl}$ & $p$ value \\
\hline Systolic blood pressure, $\mathrm{mmHg}$ & 0.99 & $0.965-1.020$ & 0.678 \\
\hline Diastolic blood pressure, $\mathrm{mmHg}$ & 1.04 & $0.994-1.080$ & 0.094 \\
\hline Serum sodium level, mEq/L & 1.14 & $0.878-1.480$ & 0.326 \\
\hline Serum potassium level, mEq/L & 0.28 & $0.085-0.907$ & 0.034 \\
\hline C-serum osmolality, mOsm/L & 1.09 & $0.981-1.210$ & 0.109 \\
\hline \multicolumn{4}{|l|}{ Total urine volume } \\
\hline Water intake (BL to day 1), mL & 1.00 & $1.000-1.000$ & 0.095 \\
\hline
\end{tabular}

\section{Characteristics of patients with hypernatremia in those with low potassium level at baseline}

There were no significant differences in the renin activity and aldosterone level and medications with loop diuretics, angiotensin-converting enzyme inhibitors/angiotensin receptor blockers, and aldosterone antagonists between patients with serum potassium level $\leq 3.8 \mathrm{mEq} / \mathrm{L}$ at baseline with and without hypernatremia (Table 5). However, patients with hypernatremia exhibited significantly higher diastolic pressure on admission day $(p=0.004)$ among those with serum potassium level $\leq 3.8 \mathrm{mEq} / \mathrm{L}$ (Table 5 ). The ratio of aldosterone level to renin activity tended to be high in patients with hypokalemia with hypernatremia.

\section{Discussion}

Hypernatremia in the initial three days of hospitalization after tolvaptan administration in addition to standard therapy, including carperitide infusion, in patients with ADHF was associated with low serum potassium level at baseline in the multivariate regression analysis. Among patients with serum potassium level $\leq 3.8 \mathrm{mEq} / \mathrm{L}$, the cutoff value by receiver operating

Table 3 Water balance in patients with and without hypernatremia

\begin{tabular}{llll}
\hline & With hypernatremia & Without hypernatremia & $\boldsymbol{p}$ value \\
\hline Total urine volume (BL to day 3), $\mathrm{mL}$ & $10,610 \pm 5327$ & $8816 \pm 4062$ & 0.096 \\
Total water intake (BL to day 3), $\mathrm{mL}$ & $3530 \pm 1643$ & $2799 \pm 1591$ & 0.089 \\
Urine volume - water intake, $\mathrm{mL}$ & & & $1507 \pm 1189$ \\
$\quad$ From BL to day 1 & $2188 \pm 1583$ & $1700 \pm 1480$ & 0.036 \\
From days 1 to 2 & $1978 \pm 1589$ & $1389 \pm 1221$ & 0.465 \\
From days 2 to 3 & $1681 \pm 2051$ & 0.427 \\
\hline
\end{tabular}

$B L$ baseline 

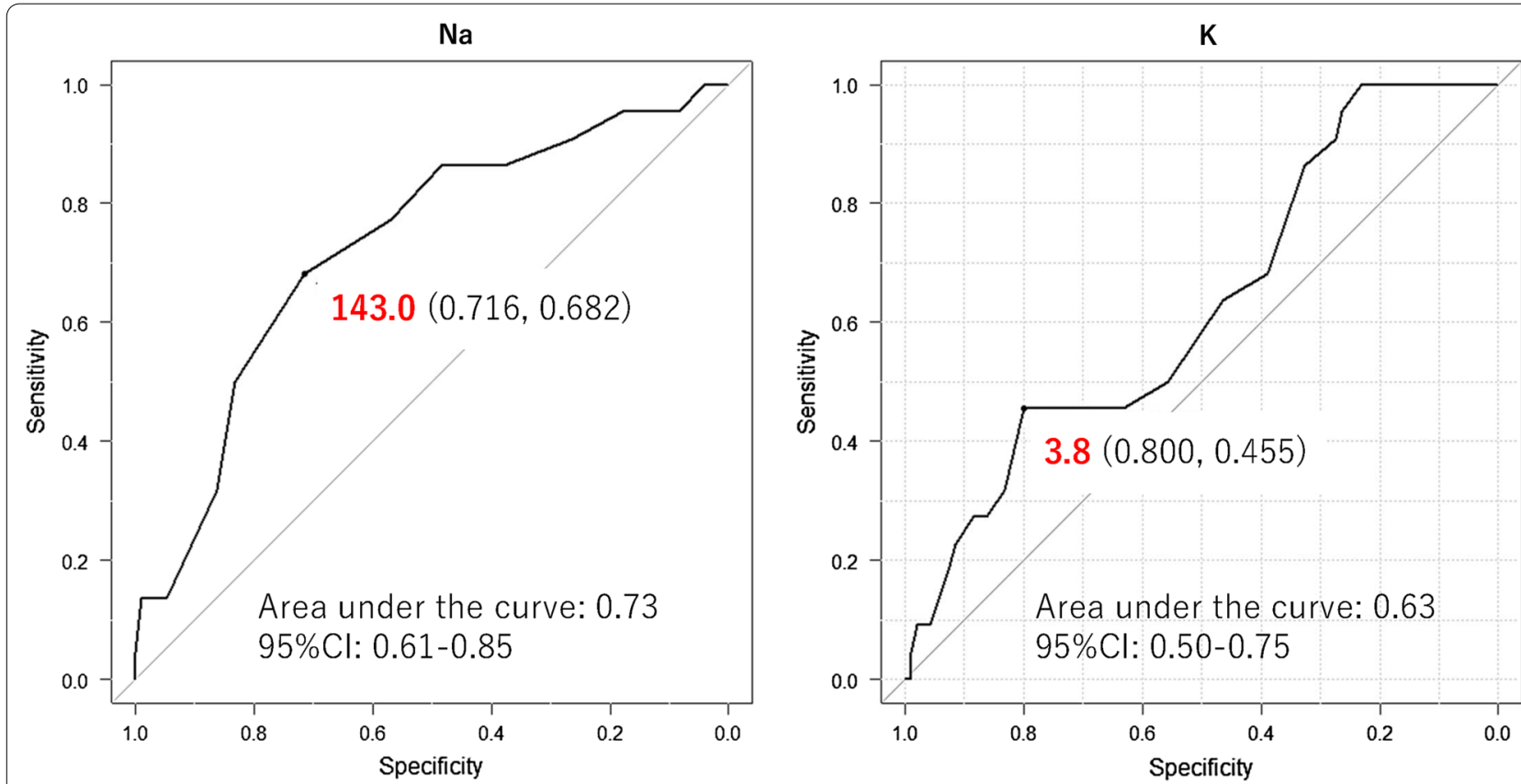

Fig. 1 The cutoff serum sodium and potassium levels at baseline by receiver operating characteristic curve analysis in patients with decompensated heart failure with hypernatremia in the initial 3 days of hospitalization after tolvaptan administration in addition to standard therapy, including carperitide infusion

Table 5 Baseline characteristics of patients whose baseline potassium level $\leq 3.8 \mathrm{mEq} / \mathrm{L}$ stratified based on the presence or absence of hypernatremia

\begin{tabular}{llll}
\hline & With hypernatremia $(\mathbf{n = 1 0})$ & Without hypernatremia $(\mathbf{n}=\mathbf{1 9})$ & $\boldsymbol{p}$ value \\
\hline Serum sodium, mEq/L & $144 \pm 2$ & $142 \pm 2$ & 0.080 \\
ACEI/ARB, \% & 60 & 53 & 0.717 \\
MRA, \% & 30 & 26 & 0.840 \\
Loop diuretics, \% & 50 & 58 & 0.697 \\
FENa, \% & $2.3 \pm 2.1$ & $3.7 \pm 5.6$ & 0.624 \\
FEUN, \% & $42.0 \pm 8.5$ & $39.8 \pm 9.0$ & 0.665 \\
FEK, \% & $12.6 \pm 7.2$ & $14.7 \pm 10.1$ & 0.684 \\
TTKG & $3.6 \pm 1.8$ & $4.0 \pm 1.6$ & 0.677 \\
PRA, $n g / m L / h$ & $0.5 \pm 0.4$ & $3.5 \pm 5.9$ & 0.241 \\
PAC, pg/mL & $57.3 \pm 31.0$ & $80.1 \pm 53.6$ & 0.353 \\
PAC/PRA & $186 \pm 211$ & $114 \pm 112$ & 0.353 \\
Systolic blood pressure, $\mathrm{mmHg}$ & $157 \pm 22$ & $139 \pm 22$ & 0.050 \\
Diastolic blood pressure, $\mathrm{mmHg}$ & $97 \pm 20$ & $75 \pm 17$ & 0.004
\end{tabular}

$A C E l$ angiotensin-converting enzyme inhibitor, $A R B$ angiotensin receptor blocker, $M R A$ mineralocorticoid receptor antagonist, $F E N a$ fractional excretion of sodium, FEUN fractional excretion of urea nitrogen, FEK fractional excretion of potassium, TTKG trans-tubular K gradient, PRA plasma renin activity, PAC plasma aldosterone concentration, PAC/PRA, ratio of plasma aldosterone concentration to plasma renin activity

characteristic curve analysis, those with hypernatremia related to tolvaptan treatment showed significantly higher diastolic blood pressure on admission day.
Tolvaptan and renin-angiotensin-aldosterone system (RAAS)

Secondary aldosteronism with HF and loop diuretic therapy may be attributed to hypokalemia [15]. Aldosterone stimulates sodium reabsorption and potassium excretion via $\mathrm{Na}+-\mathrm{K}+$ ATPase in the renal tubules 
of these patients, leading to hypokalemia. However, tolvaptan inhibits angiotensin II-induced increases in aldosterone production via a V2 receptor-independent pathway in vitro [16]. Furthermore, treatment with tolvaptan plus natriuretic peptide does not activate RAAS [17] and prevents an increase in aldosterone levels compared to that with natriuretic peptide only [18]. In patients with hypokalemia at baseline, those with hypernatremia exhibited higher diastolic blood pressure, although there was no difference in medications with angiotensin-converting enzyme inhibitors/angiotensin II receptor blockers, aldosterone antagonists, and loop diuretics in those without hypernatremia in this study. The ratio of aldosterone level to renin activity tended to be higher in patients with hypokalemia with hypernatremia. These results suggest that the inhibitory effects of RAAS by RAAS inhibitor treatment were less or breakthrough phenomena of RAAS occurred in patients with hypokalemia with hypernatremia than in those without hypernatremia.

There are individual differences in the inhibitory extents of RAAS by RAAS inhibitor treatment, and the frequency of use of aldosterone blockade (approximately $20 \%$ ) was lower in this study compared to those in other studies (approximately 40\%) [11, 12]. The use of loop diuretics results in the inhibition of sodium reabsorption, but aldosterone blockade may be insufficient in patients with hypokalemia despite RAAS inhibitor treatment in consideration with higher blood pressure. Moreover, hypokalemia reduces urine concentration and induces an increase in urine volume, thus resulting in hypernatremia in addition to the effect of tolvaptan. These findings may indicate the pathophysiologically more severe state of HF in patients with hypokalemia with hypernatremia, which could be clarified by a further study examining prognosis in these patients.

It is well known that tolvaptan can decrease body weight and increase the sodium level in patients who are with ADHF [19]. We used the criteria of hypernatremia as sodium level $\geq 148 \mathrm{mEq} / \mathrm{L}$ (out of normal range in our hospital) in the initial three days of hospitalization, which was different from that in the previous study showing the risk factors for tolvaptan-induced hypernatremia $(\geq 147 \mathrm{mEq} / \mathrm{L}[10] ; \geq 150 \mathrm{mEq} / \mathrm{L}[11,12])$. The incidence of hypernatremia was higher (19\%) in this study than those in previous studies, resulting from the threshold of hypernatremia $[11,12]$ or included patients with liver cirrhosis $(0.2 \%)$ [20]. In patients with liver cirrhosis, tolvaptan-induced hypernatremia was not related to hypokalemia, possibly because almost all patients with liver cirrhosis were administered spironolactone [10]. These findings strongly suggest that aldosteronerelated factors may be involved in hypernatremia and hypokalemia of patients treated with tolvaptan. The combined use of tolvaptan and adequate RAAS inhibitors may be recommended to prevent hypernatremia in loop diuretic-refractory ADHF.

\section{Limitations}

Some limitations are to be noted in this study: It is a single-center study; a study showing additive effect of tolvaptan in association with standard therapy, including carperitide infusion, in patients with ADHF; and not a dose-finding study. The routine use of carperitide is not recommended as a first-line vasodilator for elderly patients with ADHF [21]. Although urine examination result, such as urine osmolality, was used to predict response to tolvaptan [22], we did not observe differences in urine factors, such as urine osmolality and urine sodium/creatinine ratio, between patients with and without hypernatremia in this study. Some important clinical data such as echocardiographic indices were lacking for better, cautious understanding of the study results.

\section{Conclusion}

In tolvaptan treatment combined with standard therapy in patients with ADHF, serum potassium level $\leq 3.8 \mathrm{mEq} / \mathrm{L}$ at baseline may be a determinant factor for the development of hypernatremia. Among patients with hypokalemia, those with higher diastolic blood pressure on admission may be carefully managed to prevent hypernatremia, possibly because of the involvement of aldosterone-related factors.

\section{Abbreviations}

ADHF: Acute decompensated heart failure; BNP: Brain natriutetic peptide; HF: Heart failure; RAAS: Renin-angiotensin-aldosterone system; UCr: Urine creatinine; UNa: Urine sodium; UUN: Urine urea nitrogen.

\section{Acknowledgements \\ None.}

Authors' contributions

Conception and design of the study, or acquisition of, or analysis and interpretation of data: HF, YS, TM, HI, KU, SI, KM, KU. Drafting the article or revising it critically for important intellectual content: HF, KT, SH. Final approval of the version to be submitted: All authors have read and approved the submission of the manuscript.

\section{Funding}

None.

\section{Availability of data and materials}

The raw data may be made available upon reasonable request from the corresponding author.

\section{Ethics approval and consent to participate}

This study complied with the tenets of the Declaration of Helsinki. Because our study was performed in a retrospective manner, a local ethics committee (Ethics Committee of Yao Municipal Hospital) ruled that no formal ethics 
approval or consent was required in this study. The director of our hospital granted permission to access and use the raw data.

\section{Consent for publication}

Not Applicable.

\section{Competing interests}

The authors have no financial or other relations that could lead to a conflict of interest.

Received: 7 July 2020 Accepted: 22 October 2020

Published online: 29 October 2020

\section{References}

1. Yamamura $Y$, Nakamura S, Itoh S, Hirano T, Onogawa T, Yamashita T, Yamada Y, Tsujimae K, Aoyama M, Kotosai K, Ogawa H, Yamashita H, Kondo K, Tominaga M, Tsujimoto G, Mori T. OPC-41061, a highly potent human vasopressin V2-receptor antagonist: pharmacological profile and aquaretic effect by single and multiple oral dosing in rats. J Pharmacol Exp Ther. 1998;287:860-7.

2. Schrier RW, Gross P, Gheorghiade M, Berl T, Verbalis JG, Czerwiec FS, Orlandi C, Investigators SALT. Tolvaptan, a selective oral vasopressin V2-receptor antagonist, for hyponatremia. N Engl J Med. 2006;355:2099-112.

3. Gassanov N, Semmo N, Semmo M, Nia AM, Fuhr U, Er F. Arginine vasopressin (AVP) and treatment with arginine vasopressin receptor antagonists (vaptans) in congestive heart failure, liver cirrhosis and syndrome of inappropriate antidiuretic hormone secretion (SIADH). Eur J Clin Pharmacol. 2011;67:333-46.

4. Wang C, Xiong B, Cai L. Effects of tolvaptan in patients with acute heart failure: a systematic review and meta-analysis. BMC Cardiovasc Disord. 2017;17:164. https://doi.org/10.1186/s12872-017-0598-y.

5. Wu MY, Chen TT, Chen YC, Tarng DC, Wu YC, Lin HH, Tu YK. Effects and safety of oral tolvaptan in patients with congestive heart failure: a systematic review and network meta-analysis. PLoS ONE. 2017;12:e0184380.

6. Alskaf E, Tridente A, Al-Mohammad A. Tolvaptan for heart failure, systematic review and meta-analysis of trials. J Cardiovasc Pharmacol. 2016:68:196-203.

7. Gunderson EG, Lillyblad MP, Fine M, Vardeny O, Berei TJ. Tolvaptan for volume management in heart failure. Pharmacotherapy. 2019;39:473-85.

8. Darmon M, Timsit JF, Francais A, Nquile-Makao M, Adrie C, Cohen Y, Garrouste-Orgeas M, Goldgran-Toledano D, Dumenil AS, Jamali S, Cheval C, Allaouchiche B, Souweine B, Azoulay E. Association between hypernatremia acquired in the ICU and mortality: a cohort study. Nephrol Dial Transplant. 2010;25:2510-5.

9. Funk GC, Lindner G, Druml W, Metnitz B, Schwarz C, Bauer P, Metnitz PG. Incidence and prognosis of dysnatremias present on ICU admission. Intensive Care Med. 2010;36:304-11.

10. Hirai K, Shimomura T, Moriwaki H, Ishii H, Shimoshikiryo T, Tsuji D, Inoue K, Kadoiri T, Itoh K. Risk factors for hypernatremia in patients with short- and long-term tolvaptan treatment. Eur J Clin Pharmacol. 2016;72:1177-83.

11. Kinugawa K, Sato N, Inomata T, Shimakawa T, Iwatake N, Mizuguchi K. Efficacy and safety of tolvaptan in heart failure patients with volume overload - an interim result of post-marketing surveillance in Japan. Circ J. 2014;78:844-52
12. Kinugawa K, Sato N, Inomata T, Yasuda M, Shibasaki Y, Shimakawa T. Novel risk score efficiently prevents tolvaptan-induced hypernatremic events in patients with heart failure. Circ J. 2018;82:1344-50.

13. Gheorghiade M, Niazi I, Ouyang J, Czerwiec F, Kambatashi J, Zampino M, Orlandi C, Tolvaptan Investigators. Vasopressin V2-receptor blockade with tolvaptan in patients with chronic heart failure: results from a doubleblind, randomized trial. Circulation. 2003;107:2690-6.

14. Konstam MA, Gheorghiade M, Burnett JC Jr, Grinfeld L, Maggioni AP, Swedberg K, Udelson JE, Zannad F, Cook T, Ouyang J, Zimmer C, Orlandi C, Efficacy of Vasopressin Antagonism in Heart Failure Outcome Study With Tolvaptan (EVEREST) Investigators. Effects of oral tolvaptan in patients hospitalized for worsening heart failure: the ELEVEST outcome trial. JAMA. 2007;297:1319-31.

15. Kinugawa K, Inomata T, Sato N, Yasuda M, Shimakawa T, Bando K, Mizuguchi K. Effectiveness and adverse events of tolvaptan in octogenarians with heart failure interim analyses of Samsca Post-Marketing Surveillance In Heart failurE (SMILE Study). Int Heart J. 2015;56:137-43.

16. Ali F, Dohi K, Okamoto R, Katayama K, Ito M. Novel molecular mechanisms in the inhibition of adrenal aldosterone synthesis: action of tolvaptan via vasopressin $\mathrm{V} 2$ receptor-independent pathway. Br J Pharmacol. 2019;176:1315-27.

17. Jujo K, Saito K, Ishida I, Furuki Y, Kim A, Suzuki Y, Sekiguchi H, Yamaguchi J, Ogawa H, Hagiwara N. Randomized pilot trial comparing tolvaptan with furosemide on renal and neurohumoral effects in acute heart failure. ESC Heart Fail. 2016;3:177-88.

18. Costello-Boerrigter LC, Boerrigter G, Cataliotti A, Harty GJ, Burnett JC Jr. Renal and anti-aldosterone actions of vasopressin-2 receptor antagonism and B-type natriuretic peptide in experimental heart failure. Circ Heart Fail. 2010;3:412-9.

19. Ma G, Ma X, Wang G, Teng W, Hui X. Effect of tolvaptan add-on therapy in patients with acute heart failure: meta-analysis on randomized controlled trials. BMJ Open. 2019;9:e025537. https://doi.org/10.1136/bmjopen-2018025537.

20. Sakaida I, Terai S, Kurosaki M, Yasuda M, Okada M, Bando K, Fukuta Y. Effectiveness and safety of tolvaptan in liver cirrhosis patients with edemainterim results of Samsca posT-mARkeTing surveillance in liver cirrhosis (STATR study). Hepatol Res. 2017;47:1137-46.

21. Nagai T, Iwakami N, Nakai M, Nishimura K, Sumita Y, Mizuno A, Tsutsui H, Ogawa H, Anzai T, Investigators JROAD-DPC. Effect of intravenous carperitide versus nitrates as first-line vasodilators on in-hospital outcomes in hospitalized patients with acute heart failure: Insight from a nationwide claim-based database. Int J Cardiol. 2019;280:104-9.

22. Imamura T, Kinugawa K, Shiga T, Kato N, Muraoka H, Minatsuki S, Inaba T, Maki H, Hatano M, Yao A, Kyo S, Nagai R. Novel criteria of urine osmolality effectively predict response to tolvaptan in decompensated heart failure patients: Association between non-responders and chronic kidney disease. Circ J. 2013;77:397-404.

\section{Publisher's Note}

Springer Nature remains neutral with regard to jurisdictional claims in published maps and institutional affiliations. 